\title{
Duration of high-dose aspirin therapy does not affect long-term coronary artery outcomes in Kawasaki disease
}

\author{
Karl Migally ${ }^{1}$, Elizabeth A Braunlin ${ }^{1}$, Lei Zhang ${ }^{2}$ and Bryce A Binstadt ${ }^{3}$
}

BACKGROUND: High-dose aspirin (HDA) is used with intravenous immunoglobulin (IVIg) in Kawasaki disease (KD). Practice regarding HDA varies, and it is unclear whether HDA duration affects the long-term course.

METHODS: We retrospectively studied KD patients at our hospital for over 10 years. Patients were categorized as having received HDA for $0,1-7$, or $>7$ days. Primary outcome was the maximum coronary Z-score at diagnosis and follow-up; secondary outcomes included inflammatory markers.

RESULTS: One hundred and three patients had HDA duration documented, of which 35 patients had coronary artery abnormalities (CAAs) at diagnosis. There was no difference in demographics or inflammatory markers between the HDA groups, and no difference in HDA duration between patients with or without CAAs. Seventeen patients received no HDA; they had longer illness and defervescence duration before diagnosis, and were less likely to receive IVIg. For CAAs, multivariate regression revealed that HDA duration did not predict the coronary Z-score at 9-15 months. Higher Z-score at diagnosis was associated with higher $Z$-score at 915 months.

CONCLUSION: The only factor associated with coronary Zscore at 9-15 months was the Z-score at diagnosis. At our institution, longer illness and defervescence duration and the lack of IVlg administration were associated with not administering HDA. HDA duration did not affect the clinically relevant outcomes, particularly CAA persistence.

K awasaki disease $(\mathrm{KD})$ is an acute vasculitis syndrome first described by Dr Tomisaku Kawasaki in 1967 (1). It most commonly affects children under the age of 5 years and is typically self-limited. Its classic symptoms include high fever and signs of generalized inflammation of the skin, eyes, mouth, cervical lymph nodes, and palms and soles (2). The vasculitis affects medium-sized blood vessels, most notably coronary artery abnormalities (CAAs), and can cause chronic alterations in the coronary circulation. CAAs occur in up to $25 \%$ of untreated patients (3), with the most serious consequence being death from acute coronary artery thrombosis (4).
Intravenous immunoglobulin (IVIg) was first shown in 1984 to reduce the incidence of CAAs in KD patients (5). A number of dosing regimens were studied, and in 1991, the most effective of those was shown to be a single infusion of $2 \mathrm{~g} / \mathrm{kg}$ IVIg aspirin administered over $12 \mathrm{~h} \mathrm{(6).} \mathrm{This} \mathrm{dose} \mathrm{was}$ instituted in the 1993 American Heart Association (AHA) scientific statement on KD (7) and has been standardly used since then.

Acetylsalicylic acid or aspirin was first proposed as a therapeutic option for $\mathrm{KD}$, given both its anti-inflammatory and anti-platelet effects, when thrombocytosis was recognized as a key feature of the disease 6 years after its initial description (8). At moderate $(30-50 \mathrm{mg} / \mathrm{kg}$ per day) and high $(80-100 \mathrm{mg} / \mathrm{kg}$ per day) doses, aspirin exerts antiinflammatory effects, whereas at lower doses $(3-5 \mathrm{mg} / \mathrm{kg}$ per day), the predominant effect is that of anti-platelet. The initial recommended dose of aspirin in the acute phase of $\mathrm{KD}$ in Japan was $30 \mathrm{mg} / \mathrm{kg}$ per day $(9,10)$. Simultaneously, data published in the United States demonstrated that malabsorption of aspirin may occur in the acute phase, necessitating higher doses to achieve clinical effects $(11,12)$. This notion was supported by the data indicating that aspirin doses of $80-$ $100 \mathrm{mg} / \mathrm{kg}$ per day could shorten the duration of fever (4) and, when compared to no aspirin therapy, were associated with a decreased incidence of CAAs (13).

These early data on the effect of aspirin on CAAs were questioned in 1992 when a multicenter study demonstrated no difference in the incidence of CAAs between patients treated with IVIg and high-dose aspirin (HDA), defined in that trial as $100 \mathrm{mg} / \mathrm{kg}$ per day vs. those treated with IVIg and low-dose aspirin (5-10 mg/kg per day) (14). The same conclusion was reached by a meta-analysis that showed no difference in the incidence of CAAs at 30 or 60 days after disease onset between the low-dose aspirin or HDA groups, with HDA being defined as $>80 \mathrm{mg} / \mathrm{kg}$ per day (15). A subsequent meta-analysis showed that the prevalence of CAAs was inversely related to the total dose of IVIg but was independent of the aspirin dose used (16).

With respect to the effects of aspirin on the duration of fever, which can influence the length of hospitalization, a 1992 study found that HDA was associated with a shorter duration

${ }^{1}$ Division of Cardiology, University of Minnesota, Minneapolis, Minnesota; ${ }^{2}$ Department of Pediatrics, and Clinical and Translational Sciences Institute, University of Minnesota, Minneapolis, Minnesota; ${ }^{3}$ Division of Pediatric Rheumatology, University of Minnesota, Minneapolis, Minnesota. Correspondence: Bryce A. Binstadt (binstadt@umn.edu)

Received 3 July 2017; accepted 2 March 2018; advance online publication 2 May 2018. doi:10.1038/pr.2018.44 
of fever (14); however, a more recent comparison of HDA and low-dose aspirin in the treatment of $\mathrm{KD}$ showed no difference in fever duration between the two groups (17).

The conflicting evidence regarding the effects of HDA on CAA incidence and fever duration is reflected in the guidelines for the treatment of KD. In 1988, a consensus statement prepared by North American participants of the Third International Kawasaki Disease Symposium recommended HDA of $80-100 \mathrm{mg} / \mathrm{kg}$ per day for 14 days in the management of KD (18). Five years later, the AHA statement on KD recommended HDA until the patient was afebrile and acknowledging; however, some clinicians recommend HDA until the 14th day of the illness (7). The 2004 and recently published 2017 AHA statements on KD once again recognized the differences in practices regarding HDA dosing among institutions $(19,20)$. Both statements specifically note that HDA "does not appear to lower the frequency of the development of coronary abnormalities," based on evidence evaluating the effect of HDA on CAAs at early follow-up 30 and 60 days after diagnosis $(15,21)$. The 2017 AHA statement recommends that "administration of moderate- $(30-50 \mathrm{mg} / \mathrm{kg}$ per day) to high-dose $(80-100 \mathrm{mg} / \mathrm{kg}$ per day) aspirin is reasonable until the patient is afebrile, although there is no evidence that it reduces coronary artery aneurysms" (20). Furthermore, there are no data to indicate the superiority of either the $30-50 \mathrm{mg} / \mathrm{kg}$ per day dose used in Japan and Europe or the $80-100 \mathrm{mg} / \mathrm{kg}$ per day dose used in the United States (20).

The purpose of the present study was to evaluate a single center's experience of demographic and illness factors that may affect the clinician practices regarding HDA duration prescribed in acute $\mathrm{KD}$, as well as the impact of HDA duration on key clinical outcomes, including long-term status of CAAs up to 15 months after diagnosis, IVIg resistance, duration of fever, and normalization of inflammatory markers.

\section{METHODS}

A retrospective study was performed at the University of Minnesota Masonic Children's Hospital, a tertiary referral center. Medical records of all children with an International Classification of Diseases- 9 diagnosis of KD between September 2006 and December 2015 were reviewed. Of 126 patients who met the criteria of acute $\mathrm{KD}, 103$ patients had HDA duration documented and were included in the study. This chart review was approved by the University of Minnesota Institutional Review Board.

During the decade of this study, there was no standardized approach to determine the duration of HDA therapy at our institution. Because numerous providers cared for these patients, there was variability in practice regarding HDA duration. The decision to change from HDA to low-dose aspirin appeared to be influenced by laboratory markers of inflammation or the presence of $\mathrm{CAA}$, but the rationale for the timing of the change was not clearly documented in several cases.

Patients were categorized as having received one of three durations of HDA: $0,1-7$, or $>7$ days. Demographic and illness variables compared among these three groups included age, sex, race, days of illness before diagnosis, and classic vs. incomplete $\mathrm{KD}$ status. Fever was defined as a temperature of $38^{\circ} \mathrm{C}$ or higher. Criteria for the classic and incomplete KD were defined according to the 2017 AHA

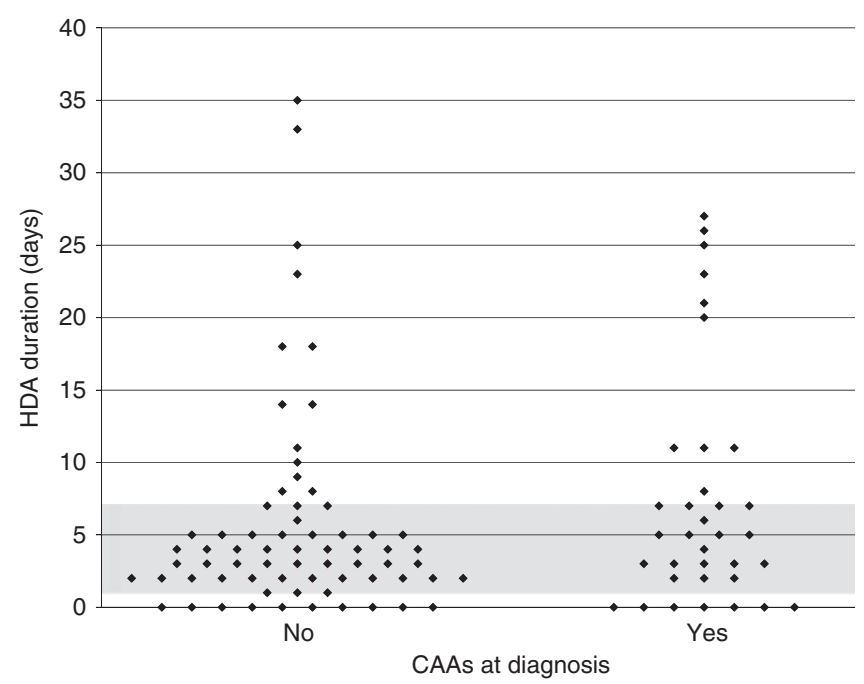

Figure 1. Categorization of study subjects based on presence or absence of coronary artery abnormalities (CAAs) at diagnosis and the duration of high-dose aspirin (HDA) therapy. Comparison of HDA duration between patients without CAAs at diagnosis (left) and patients with CAAs at diagnosis (right). Each point indicates one patient. Among the 1-7-day HDA group, the days of HDA were a mean of 3.75, median 3 , with an interquartile range (IQR) of $2-5$ days. Among the $>7$ days HDA group, the days of HDA were a mean of 17.78 , median 18 , and IQR $11-25$ days.

statement on KD (20). Coronary artery dimensions were determined by transthoracic echocardiography and were recorded as body surface area-adjusted $Z$-scores (22). The primary outcome measure was the maximum coronary artery $Z$-score. This measure, along with the secondary outcome measures of C-reactive protein (CRP) and platelet count were compared at the time of diagnosis, at 4-8 weeks after diagnosis, and at 9-15 months after diagnosis. CAAs were defined as the maximum coronary artery $Z$-score greater than or equal to 2.5 (2). Other secondary outcomes recorded included days of fever after diagnosis and IVIg resistance. IVIg-resistant patients were defined as those who received more than one dose of $2 \mathrm{~g} / \mathrm{kg}$ of IVIg and/or additional therapies, including corticosteroids, biologic therapeutics, or other immunosuppressive agents, given at least $36 \mathrm{~h}$ since the end of the first IVIg infusion.

Statistical analysis was performed using the SAS software package (version 9.3; SAS Institute Inc., Cary, NC). $P$ values were calculated using one-way analysis of variance for continuous variables and Fisher's exact test for categorical variables. A $P$ value of $<0.05$ was considered statistically significant. The statistical significance of multiple comparisons was determined using the Bonferroni adjustment (23). To assess the impact of HDA duration on longitudinal variables, a linear mixed model was used to account for the withinsubject correlation with adjustment for age at diagnosis, sex, and IVIg resistance. A linear regression model with the same adjustments was used to determine the predictors of coronary artery $Z$-score at 9-15 months in patients who had developed CAAs at diagnosis.

\section{RESULTS}

\section{Patient Characteristics}

Of 126 patients diagnosed with acute $\mathrm{KD}$ in the study time frame, 103 patients had HDA duration documented and were included in the study. Among the included patients, 63 (61\%) were male, 68 (66\%) were white, and $72(70 \%)$ were under the age of 5 years. Seventeen patients (17\%) received 0 days of HDA, 63 patients (61\%) received 1-7 days of HDA, and 23 
patients (22\%) received $>7$ days of HDA. Of the 103 study patients, 35 (34\%) patients had CAAs at diagnosis (Figure 1). Only two of those patients had isolated left main coronary artery dilation; this is a location where $Z$-scores should be interpreted with caution because of frequent anatomic variations (20); however, reviews of the echocardiograms in our two patients indicated obviously dilated vessels (maximum $Z$-score of 44.24 in one case and 3.54 in the other, with borderline ectasia of the left anterior descending artery in the latter).

\section{Clinical Correlates of HDA Duration}

All patients. Within the overall study population, three factors correlated with the duration of HDA prescribed: illness duration before diagnosis, duration of defervescence before diagnosis, and administration of IVIg (Table 1). Specifically, illness duration and the duration of defervescence before diagnosis were both longer in the 0-day HDA group than in the other two groups $(P<0.001$ for both variables). Among the 17 patients in the 0-day HDA group, 5 had fever at the time of diagnosis, and all 5 of them received IVIg. Overall, a significantly lower proportion of patients in the 0-day HDA group received IVIg compared with the other groups (68\% vs. $98 \%$ and $100 \%, P<0.001)$. All but one patient who received any HDA received IVIg, whereas 6 of 17 patients in the 0 -day HDA group (35\% of group) did not receive IVIg. It is unusual for a patient to receive HDA but not IVIg; in this case, the primary care provider started HDA for KD based on the recurrence of a peeling rash several days after an initial 5-day period of fever and rash. However, the consulting cardiologist did not recommend IVIg because the fever had resolved and the echocardiogram was normal; the HDA was then discontinued.

There was no association between patient age, sex, or race, and the duration of HDA in the overall study population. The proportions of patients with classic vs. incomplete $\mathrm{KD}$ and with CAAs at the time of diagnosis were also not different between the three HDA groups (Table 1). The CRP at diagnosis was not associated with the duration of HDA. There was a statistically significant difference in platelet count at diagnosis between the three HDA groups, with the 0 days of HDA group having the highest value, followed by the $>7$-day group, and then the 1-7-day group (Table 1).

Patients without CAAs at diagnosis. Table 2 contains data regarding the subgroup of 68 patients without CAAs at the time of diagnosis. Within this group, 10 patients $(15 \%)$ received 0 days of HDA, 45 (66\%) received 1-7 days of HDA, and 13 $(19 \%)$ received $>7$ days of HDA. Four of the 10 patients who did not receive HDA also did not receive IVIg, whereas only one patient who received HDA did not receive IVIg (described above); pairwise comparison demonstrated a significant difference between the 0-day HDA group and the 1-7-day HDA group for IVIg administration (60\% vs. $98 \%, P=0.003$ ). As with the overall study population, illness duration and the duration of defervescence before diagnosis were longer in the 0 -day HDA group compared within the other two groups
$(P<0.001$ for both variables). The average age at diagnosis was higher in the 0-day HDA group (9.01 years) compared with in the other two groups ( 3.74 and 2.87 years, $P<0.001$ ), whereas the platelet count at diagnosis was higher in the $>7$-day HDA group $\left(522.5 \times 10^{9} / \mathrm{L}\right)$ compared with the other two groups $\left(324.3 \times 10^{9} / \mathrm{L}\right.$ and $\left.352.7 \times 10^{9} / \mathrm{L}, P<0.001\right)$. There was no association between patient sex, race, or KD status, and the duration of HDA prescribed.

Patients with CAAs at diagnosis. Table 3 contains data regarding the subgroup of 35 patients with CAAs at the time of diagnosis. Despite the presence of CAAs at diagnosis, seven patients (20\%) received 0 days of HDA; 18 (51\%) received 1-7 days of HDA, and 10 (29\%) received $>7$ days of HDA. Two of the seven patients who did not receive HDA also did not receive IVIg, whereas $100 \%$ of patients who received HDA also received IVIg. As with the overall study population, illness duration was significantly longer in the 0-day HDA group (29.14 days) compared with the other two HDA groups (9.35 and 11.70 days, $P<0.001$ ), whereas the duration of defervescence was longer in the 0-day HDA group compared with the $1-7$-day HDA group (12.86 vs. 0.06 days, $P=0.002$ ). The fraction of female patients was higher in the 0-day HDA group (71\%) and lowest in the $>7$-day HDA group (10\%).

Similar to the entire study population, there was no association between patient age, race, or $\mathrm{KD}$ status and the duration of HDA among patients with CAAs at diagnosis. The maximum coronary artery $Z$-score value, CRP level, and platelet count at diagnosis were also not correlated with the duration of the HDA prescribed.

\section{Clinical Course and Outcomes Based on the Duration of HDA}

All patients. Among the overall study population, there was no association between the duration of HDA prescribed and IVIg resistance, fever duration after diagnosis, CRP, or platelet count at 4-8 weeks or 9-15 months (Table 1).

Patients without CAAs at diagnosis. Between the three HDA groups, univariate analysis of the 68 patients without CAAs at diagnosis (Table 2) demonstrated a higher CRP at 4-8 weeks in the 0-day HDA group compared with the 1-7-day HDA group $(0.83 \mathrm{~g} / \mathrm{dL}$ vs. $0.32 \mathrm{~g} / \mathrm{dL}, P=0.03)$ and a higher platelet count at $4-8$ weeks in the $>7$-day HDA group compared with the 0 -day HDA group $\left(417.3 \times 10^{9} / \mathrm{L}\right.$ vs. $\left.342.0 \times 10^{9} / \mathrm{L}, P=0.03\right)$. However, neither of these variables were different between the HDA groups at 9-15 months. Similar to the overall study population, there was no association between the duration of HDA prescribed and IVIg resistance, or fever duration after diagnosis. No patients without CAAs at the time of diagnosis subsequently developed CAAs.

Patients with CAAs at diagnosis. Among patients with CAAs at the time of diagnosis, CAAs were still present at the 4-8-week timepoint in 19 of 35 patients and continued to persist even at the 9-15 months timepoint in 15 of them (Table 3). Comparing the three HDA groups, univariate 
Table 1. All-patient comparison of clinical correlates and outcome variables by HDA group

\begin{tabular}{|c|c|c|c|c|c|}
\hline \multirow[t]{2}{*}{ Variable } & \multirow[b]{2}{*}{ Statistics/category } & \multicolumn{3}{|c|}{ HDA duration group } & \multirow[t]{2}{*}{$P$ value } \\
\hline & & 0 days $(N=17)$ & $1-7$ days $(N=63)$ & $>7$ days $(N=23)$ & \\
\hline Age at diagnosis (years) & Mean (SD) & $6.56(5.89)$ & $4.19(3.61)$ & $4.24(3.92)$ & 0.10 \\
\hline \multirow[t]{2}{*}{ Age range at diagnosis } & $<1$ year (\%) & $3(18)$ & $11(17)$ & $3(13)$ & 0.12 \\
\hline & $1-5$ years $(\%)$ & $5(29)$ & $34(54)$ & $16(70)$ & \\
\hline \multirow[t]{2}{*}{ Sex } & Male (\%) & $11(65)$ & $34(54)$ & $18(78)$ & 0.12 \\
\hline & Female (\%) & $6(35)$ & $29(46)$ & $5(22)$ & \\
\hline \multirow{2}{*}{ Race } & Hispanic (\%) & $2(12)$ & $8(13)$ & $1(4)$ & \\
\hline & Other (\%) & $0(0)$ & $2(3)$ & $2(9)$ & \\
\hline \multirow[t]{3}{*}{ Classic KD } & Yes (\%) & $10(59)$ & $40(69)$ & $11(50)$ & 0.27 \\
\hline & No (\%) & $7(41)$ & $18(31)$ & $11(50)$ & \\
\hline & Unknown & 0 & 5 & 1 & \\
\hline Illness duration before diagnosis (days) & Mean (SD) & $23(16.87)^{\mathrm{ab}}$ & $8.23(5)^{\mathrm{a}}$ & $10.86(5)^{\mathrm{b}}$ & $<0.001$ \\
\hline Defervescence duration before diagnosis (days) & Mean (SD) & $11.76(13.47)^{\mathrm{ab}}$ & $0.46(2.55)^{\mathrm{a}}$ & $0.24(0.70)^{b}$ & $<0.001$ \\
\hline \multirow[t]{2}{*}{ IVlg no. 1 given } & Yes (\%) & $11(65)^{\mathrm{ab}}$ & $62(98)^{\mathrm{a}}$ & $23(100)^{b}$ & $<0.001$ \\
\hline & No (\%) & $6(35)$ & $1(2)$ & $0(0)$ & \\
\hline \multirow[t]{3}{*}{ IVlg resistance } & Yes (\%) & $5(45)$ & $14(23)$ & $10(43)$ & 0.08 \\
\hline & No (\%) & $6(55)$ & $48(77)$ & $13(57)$ & \\
\hline & Unknown & 6 & 1 & 0 & \\
\hline Fever duration after diagnosis (days) & Mean (SD) & $0.13(0.50)$ & $1.16(3.30)$ & $2.41(5.09)$ & 0.14 \\
\hline CRP at $4-8$ weeks ( $\mathrm{g} / \mathrm{dL})$ & Mean (SD) & $0.69(0.58)$ & $0.32(0.22)$ & $0.66(0.61)$ & 0.05 \\
\hline CRP at $9-15$ months $(\mathrm{g} / \mathrm{dL})$ & Mean (SD) & $0.96(1.10)$ & $0.57(0.13)$ & $0.34(0.11)$ & 0.42 \\
\hline Platelet count at $4-8$ weeks $\left(\times 10^{9} / \mathrm{L}\right)$ & Mean (SD) & $319.9(127.8)$ & $358.3(115.2)$ & $394.3(110.2)$ & 0.32 \\
\hline Platelet count at $9-15$ months $\left(\times 10^{9} / \mathrm{L}\right)$ & Mean (SD) & $388.3(145.5)$ & $283.3(106.5)$ & $264.4(59.8)$ & 0.08 \\
\hline
\end{tabular}

CAA, coronary artery abnormality; CRP, C-reactive protein; HAD, high-dose aspirin; IVlg, intravenous immunoglobulin; KD, Kawasaki disease; SD, standard deviation.

a Superscripts denote pairwise comparisons of statistical significance for variables with $P$ values $<0.05$, for a significant difference between the 0-day HDA group and the 1-7day HDA group.

${ }^{b}$ Superscripts denote pairwise comparisons of statistical significance for variables with $P$ values $<0.05$, for a significant difference between the 0 -day HDA group and the $>$ 7-day HDA group.

'Superscripts denote pairwise comparisons of statistical significance for variables with $\mathrm{P}$ values $<0.05$, for no significant differences in pairwise comparison. Bonferroni's adjustment for multiple comparisons is used, whereby a $P$ values threshold for statistical significance between pairs is $0.05 /($ number of pairwise comparisons) $=0.05 / 3=0.0167$. Bold values are to highlight $P$-values $<0.05$. 


\section{Articles | Migally et al.}

Table 2. Comparison of clinical correlates and clinical outcome variables byHDA group in patients with KD and no CAAs at diagnosis

\begin{tabular}{|c|c|c|c|c|c|}
\hline \multirow[t]{2}{*}{ Variable } & \multirow[t]{2}{*}{ Statistics/category } & \multicolumn{3}{|c|}{ HDA duration group } & \multirow[t]{2}{*}{$P$ value } \\
\hline & & 0 days $(N=10)$ & $1-7$ days $(N=45)$ & $>7$ days $(N=13)$ & \\
\hline Age at diagnosis (years) & Mean (SD) & $9.01(6.37)^{\mathrm{ab}}$ & $3.74(2.99)^{\mathrm{a}}$ & $2.87(1.50)^{b}$ & $<0.001$ \\
\hline \multirow[t]{2}{*}{ Age range at diagnosis } & $<1$ year $(\%)$ & $1(10)$ & $7(16)$ & $1(7.69)$ & 0.002 \\
\hline & $1-5$ years $(\%)$ & $2(20)$ & $27(60)$ & $12(92.31)$ & \\
\hline \multirow[t]{2}{*}{ Sex } & Male (\%) & $9(90)$ & $23(51)$ & $9(69.23)$ & 0.05 \\
\hline & Female (\%) & $1(10)$ & $22(49)$ & $4(30.77)$ & \\
\hline \multirow[t]{2}{*}{ Race } & White (\%) & $6(60)$ & $28(62)$ & $8(62)$ & 0.58 \\
\hline & Other (\%) & $0(0)$ & $2(4)$ & $1(8)$ & \\
\hline \multirow[t]{3}{*}{ Classic KD } & Yes (\%) & $6(60)$ & $33(77)$ & $5(42)$ & 0.06 \\
\hline & No (\%) & $4(40)$ & $10(23)$ & $7(58)$ & \\
\hline & Unknown & 0 & 2 & 1 & \\
\hline Illness duration before diagnosis (days) & Mean (SD) & $18.70(13.95)^{\mathrm{ab}}$ & $7.75(4.19)^{\mathrm{a}}$ & $10.09(3.78)^{b}$ & $<0.001$ \\
\hline Defervescence duration before diagnosis (days) & Mean (SD) & $11(13.31)^{\mathrm{ab}}$ & $0.63(3.04)^{\mathrm{a}}$ & $0(0)^{\mathrm{b}}$ & $<0.001$ \\
\hline CRP at diagnosis ( $\mathrm{g} / \mathrm{dL})$ & Mean (SD) & $7.18(5.27)$ & $10.31(7.84)$ & $5.23(1.60)$ & 0.18 \\
\hline \multirow{2}{*}{ IVlg resistance } & No (\%) & $4(67)$ & $38(86)$ & $9(69)$ & \\
\hline & Unknown & 4 & 1 & 0 & \\
\hline Fever duration after diagnosis (days) & Mean (SD) & $0(0)$ & $0.16(0.47)$ & $0.42(0.79)$ & 0.17 \\
\hline CRP at $4-8$ weeks $(\mathrm{g} / \mathrm{dL})$ & Mean (SD) & $0.83(0.62)^{\mathrm{a}}$ & $0.32(0.21)^{\mathrm{a}}$ & $0.52(0.33)$ & 0.03 \\
\hline CRP at 9-15 months (g/dL) & Mean (SD) & $0.48(0.20)$ & $0.63(0.18)$ & - & 0.99 \\
\hline Platelet count at $4-8$ weeks $\left(\times 10^{9} / \mathrm{L}\right)$ & Mean (SD) & $261.2(71.86)^{\mathrm{b}}$ & $342.0(104.7)$ & $417.3(110.3)^{b}$ & 0.03 \\
\hline Platelet count at $9-15$ months $\left(\times 10^{9} / \mathrm{L}\right)$ & Mean (SD) & $256.5(89.80)$ & 309.1 (95.16) & $219.0(35.36)$ & 0.42 \\
\hline
\end{tabular}

CAA, coronary artery abnormality; CRP, C-reactive protein; HAD, high-dose aspirin; IVlg, intravenous immunoglobulin; KD, Kawasaki disease; SD, standard deviation.

a Superscripts denote pairwise comparisons of statistical significance for variables with $P$ values <0.05: for a significant difference between the 0-day HDA group and the 1-7day HDA group.

${ }^{b}$ Superscripts denote pairwise comparisons of statistical significance for variables with $P$ values $<0.05$ : for a significant difference between the 0-day HDA group and the >7-day HDA group.

'Superscripts denote pairwise comparisons of statistical significance for variables with $P$ values <0.05: for a significant difference between the 1-7-day HDA group and the $>7$-day HDA group.

Bold values are to highlight $P$-values $<0.05$.

analysis demonstrated a lower incidence of persistent CAAs at 9-15 months in the 1-7-day HDA group compared with the $>7$-day HDA group ( $33 \%$ vs. $89 \%, P=0.01)$. However, multivariate analysis adjusted for age, sex, and IVIg resistance revealed no correlation between HDA duration and coronary artery dimensions at 9-15 months. Although the platelet count at 9-15 months was higher in the 0-day HDA group compared with the 1-7-day HAD group in univariate analysis 
Table 3. Comparison of clinical correlates and clinical outcome variables by HDA group in patients with Kawasaki disease (KD) and with CAAs at diagnosis

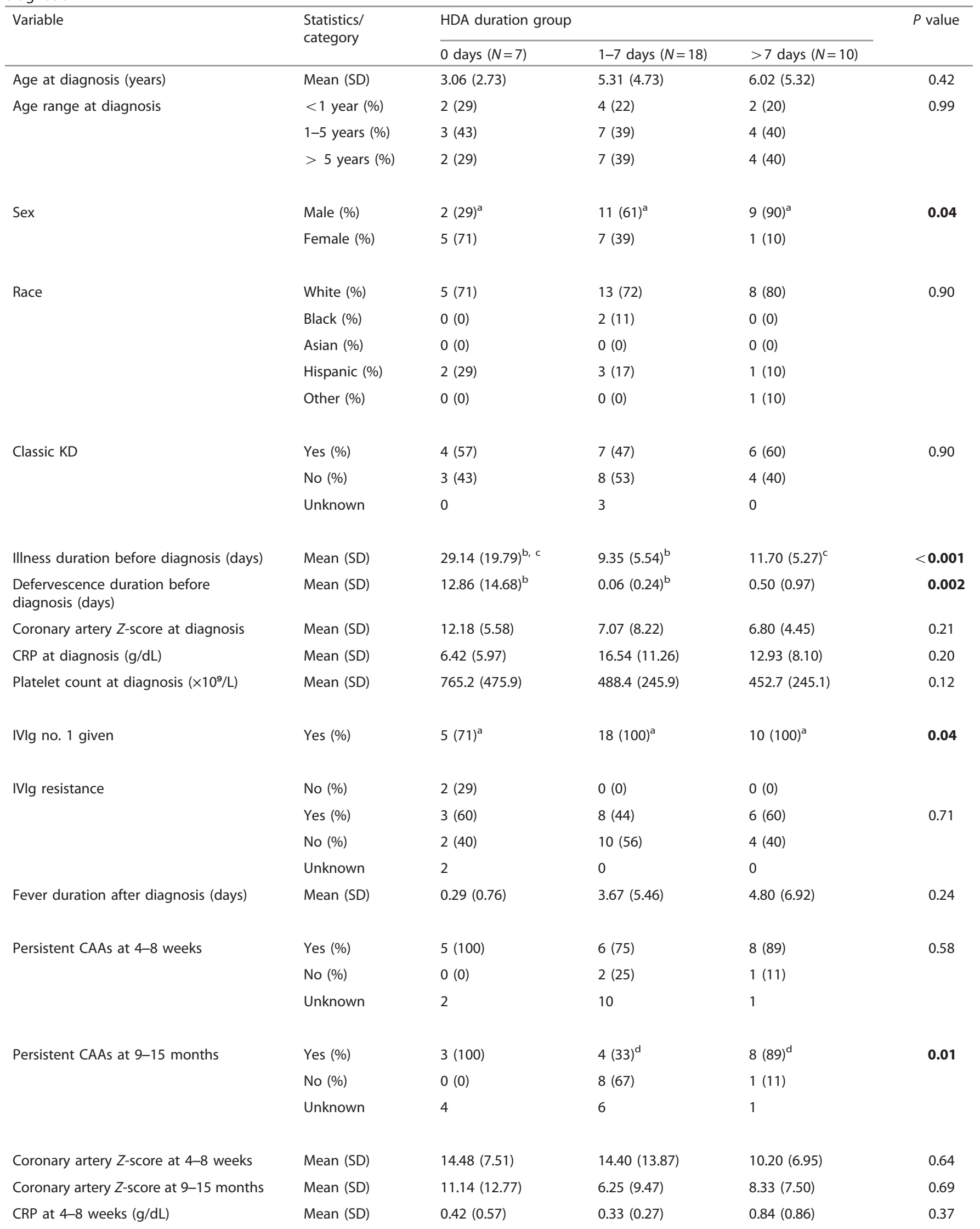


Table 3 Continued

\begin{tabular}{|c|c|c|c|c|c|}
\hline \multirow[t]{2}{*}{ Variable } & \multirow{2}{*}{$\begin{array}{l}\text { Statistics/ } \\
\text { category }\end{array}$} & \multicolumn{3}{|c|}{ HDA duration group } & \multirow[t]{2}{*}{$P$ value } \\
\hline & & 0 days $(N=7)$ & $1-7$ days $(N=18)$ & $>7$ days $(N=10)$ & \\
\hline CRP at 9-15 months (g/dL) & Mean (SD) & $1.44(1.63)$ & $0.50(0.00)$ & $0.34(0.11)$ & 0.30 \\
\hline Platelet count at $4-8$ weeks $\left(\times 10^{9} / \mathrm{L}\right)$ & Mean (SD) & $466.5(130.8)$ & $389.1(134.0)$ & $365.6(110.4)$ & 0.60 \\
\hline Platelet count at $9-15$ months $\left(\times 10^{9} / \mathrm{L}\right)$ & Mean (SD) & $454.3(123.4)^{b}$ & $250.0(118.3)^{b}$ & $279.5(60.47)$ & 0.02 \\
\hline \multicolumn{6}{|c|}{$\begin{array}{l}\text { CAA, coronary artery abnormality; CRP, C-reactive protein; HAD, high-dose aspirin; IVIg, intravenous immunoglobulin; KD, Kawasaki disease. } \\
\text { asuperscripts denote pairwise comparisons of statistical significance for variables with } P \text { values }<0.05 \text { : for no significant differences in pairwise comparison. Bonferroni's adjust- } \\
\text { ment for multiple comparisons is used, whereby a } P \text { value threshold for statistical significance between pairs is } 0.05 /(\text { number of pairwise comparisons) }=0.05 / 3=0.0167 \text {. } \\
\text { bSuperscripts denote pairwise comparisons of statistical significance for variables with } P \text { values }<0.05 \text { : for a significant difference between the } 0 \text {-day HDA group and the } 1-7 \text { - } \\
\text { day HDA group. } \\
\text { "Superscripts denote pairwise comparisons of statistical significance for variables with } P \text { values }<0.05 \text { : for a significant difference between the } 0 \text {-day HDA group and the } \\
>7 \text {-day HDA group. } \\
\text { dSuperscripts denote pairwise comparisons of statistical significance for variables with } P \text { values }<0.05 \text { : for a significant difference between the } 1-7 \text {-day HDA group and the } \\
>7 \text {-day HDA group. } \\
\text { Bold values are to highlight } P \text {-values }<0.05 \text {. }\end{array}$} \\
\hline
\end{tabular}

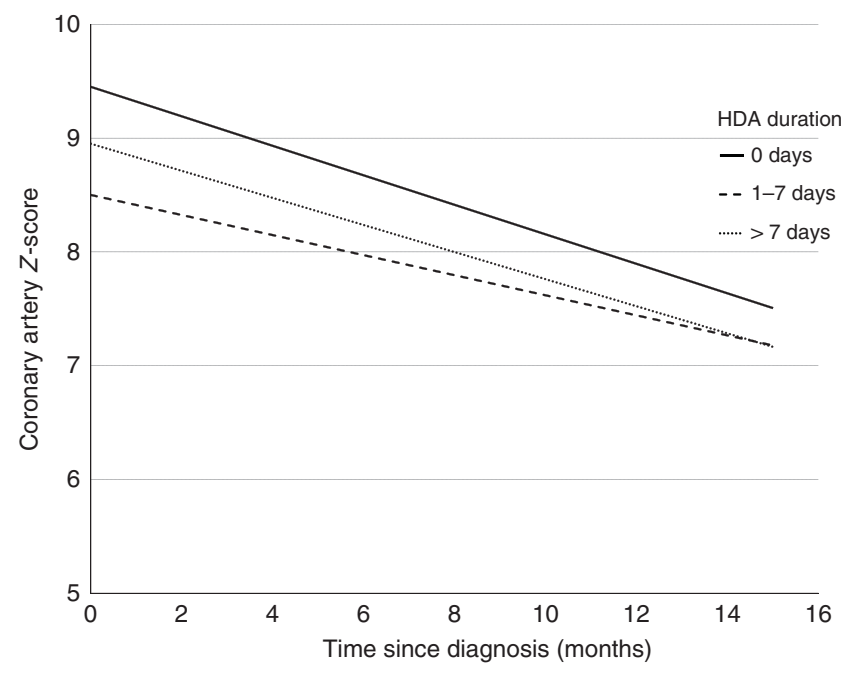

Figure 2. Rate of coronary artery Z-score decline among patients with coronary artery abnormalities (CAAs) at diagnosis, as categorized by high-dose aspirin (HDA) duration group. A linear mixed model adjusted for age, sex, and intravenous immunoglobulin (IVIg) resistance was used to generate each line, accounting for within-patient correlation and assuming that $Z$-scores were linearly associated with time.

$\left(454.3 \times 10^{9} / \mathrm{L}\right.$ vs. $\left.250 \times 10^{9} / \mathrm{L}, P=0.02\right)$, this difference did not persist in the multivariate analysis. Similar to the overall study population, there was no association between the duration of HDA prescribed and IVIg resistance, fever duration after diagnosis, or CRP at 4-8 weeks or 915 months. Longitudinal analysis adjusted for age, sex, and IVIg resistance demonstrated no difference in the rate of decline of coronary Z-score (Figure 2), CRP, or platelet count between the HDA groups.

With respect to persistence vs. resolution of CAAs at 915 months (Table 4), univariate analysis revealed that patients with persistent CAAs had longer duration of fever after the diagnosis (6.07 vs.0.67 days, $P=0.03$ ) and higher incidence of IVIg resistance ( 79 vs. $11 \%, P=0.003$ ). After adjusting for age, sex, and IVIg resistance, the only factor associated with higher coronary Z-scores at 9-15 months was higher coronary Zscores at diagnosis.

\section{DISCUSSION}

This retrospective study of 103 patients with KD was designed to understand the variations in clinical practice around the duration of HDA therapy and how these different durations affect the coronary artery outcomes beyond the acute and subacute phases of the disease. A relatively large fraction of patients in this study did not receive HDA. This group was characterized by a longer duration of illness before diagnosis and the lack of administration of IVIg. Patients who did not receive HDA represent a subset of the KD population in whom defervescence typically occurred before diagnosis as part of a prolonged illness course. Review of the medical charts of these patients indicates that the rationale behind not administering HDA was most commonly that the febrile phase of the illness had ended. However, 5 of 17 patients who had no HDA did have fever at the time of diagnosis; the reasons for not giving HDA in these five cases varied. Of note, low-dose aspirin was administered to 15 of the 17 patients who did not receive HDA; it was withheld in one patient because of the elevation of liver enzymes; and in the other, because of the practitioner's concern about precipitating Reye syndrome. Most importantly, although these factors may influence the clinical decisions regarding initiation of HDA therapy, the duration of HDA therapy itself had no impact on the clinically relevant outcome measures, particularly the late development of or persistence of CAAs and the duration of fever after diagnosis. After adjusting for IVIg resistance, a known risk factor for persistence of CAAs $(2,20)$, the only factor associated with higher coronary artery $Z$-scores at 9-15 months was higher coronary artery $Z$-scores at diagnosis. This association has been described in early (24) and late (25) follow-up, and may serve as a prognostic predictor of CAA outcomes.

Similar data exist from the 1990s and early 2000s regarding the lack of effect of HDA administration on CAA evolution $(14,15,16,21)$ and fever duration (17); however, the 2004 and 2017 AHA statements acknowledge that there is variability in practices regarding HDA dosing among institutions $(19,20)$, and our study demonstrates a wide within-institution variability in practice. In addition, these studies and newer 
Table 4. Comparison of clinical correlates and clinical outcome variables by CAA status at 9-15 months in patients with KD and CAAs at diagnosis

\begin{tabular}{|c|c|c|c|c|}
\hline Variable & Statistics/category & $\begin{array}{l}\text { Resolution of CAAs at } \\
9-15 \text { months }(N=9)\end{array}$ & $\begin{array}{l}\text { Persistence of CAAs at } \\
9-15 \text { months }(N=15)\end{array}$ & $P$ value \\
\hline Age at diagnosis (years) & Mean (SD) & $6.46(4.65)$ & $5.73(5.40)$ & 0.61 \\
\hline \multirow{2}{*}{ Age range at diagnosis } & $1-5$ years $(\%)$ & $3(33)$ & $5(33)$ & \\
\hline & $>5$ years $(\%)$ & $5(56)$ & $6(40)$ & \\
\hline Sex & Female (\%) & $3(33)$ & $4(27)$ & \\
\hline \multirow[t]{2}{*}{ Race } & White (\%) & $7(78)$ & $10(67)$ & 0.47 \\
\hline & Black (\%) & $1(11)$ & $0(0)$ & \\
\hline \multirow[t]{3}{*}{ Classic KD } & Yes (\%) & $2(29)$ & $10(71)$ & 0.16 \\
\hline & No (\%) & $5(71)$ & $4(29)$ & \\
\hline & Unknown & 2 & 1 & \\
\hline Illness duration before diagnosis (days) & Mean (SD) & $8.78(4.29)$ & $13.71(7.80)$ & 0.12 \\
\hline Defervescence duration before diagnosis (days) & Mean (SD) & $0.33(1.00)$ & $2.07(4.86)$ & 0.34 \\
\hline CRP at diagnosis (g/dL) & Mean (SD) & $15.23(11.09)$ & $12.83(7.68)$ & 0.60 \\
\hline Platelet count at diagnosis $\left(\times 10^{9} / \mathrm{L}\right)$ & Mean (SD) & $438.4(229.1)$ & $602(374.7)$ & 0.38 \\
\hline \multirow{2}{*}{ IVlg resistance } & No (\%) & $8(89)$ & $3(21)$ & \\
\hline & Unknown & 0 & 1 & \\
\hline Fever duration after diagnosis (days) & Mean (SD) & $0.67(1.00)$ & $6.07(7.10)$ & 0.03 \\
\hline CRP at $4-8$ weeks ( $\mathrm{g} / \mathrm{dL})$ & Mean (SD) & $0.41(0.32)$ & $0.66(0.74)$ & 0.75 \\
\hline CRP at 9-15 months (g/dL) & Mean (SD) & $0.50(0.00)$ & $0.33(0.09)$ & 0.07 \\
\hline Platelet count at $4-8$ weeks $\left(\times 10^{9} / \mathrm{L}\right)$ & Mean (SD) & $332.3(135.1)$ & $393.3(127.2)$ & 0.43 \\
\hline Platelet count at $9-15$ months $\left(\times 10^{9} / \mathrm{L}\right)$ & Mean (SD) & $261.3(80.56)$ & $280.1(118.8)$ & 0.87 \\
\hline
\end{tabular}

CAA, coronary artery abnormality; CRP, C-reactive protein; HDA, high-dose aspirin; IVIg, intravenous immunoglobulin; KD, Kawasaki disease.

Bold values are to highlight $P$-values $<0.05$.

ones (26) have largely evaluated the effect of HDA on shortterm outcomes in the acute and subacute phases of the disease, and so it was important to determine whether a particular HDA duration conferred measurable clinical benefit in the convalescent phase and beyond. Based on our study, there was no apparent correlation between the duration of HDA and long-term clinical outcomes, suggesting that
HDA may not be necessary in the acute management of KD. Consistent with this suggestion, Dallaire et al. (27) recently reported their findings from a retrospective study comparing HDA $(80 \mathrm{mg} / \mathrm{kg}$ per day) to low-dose aspirin $(3-5 \mathrm{mg} / \mathrm{kg}$ per day) in acute $\mathrm{KD}$; they found no difference in the risk of CAA between these two groups, and thus concluded that low-dose aspirin is not inferior to HDA for reducing CAA 
risk (27). However, another recent retrospective report found that patients who received low-dose aspirin had three times higher odds of needing IVIg re-treatment compared with those who received HDA, but with no difference in duration of hospitalization or of coronary artery aneurysms (28). Determining whether and how HDA should be used in the acute management of KD is important, because HDA has been associated with a number of adverse effects, including transaminitis (29) and rarely, Reye syndrome (30). Transaminitis was seen in two patients in our study necessitating early discontinuation of HDA, and no cases of Reye syndrome were observed.

Notably, our data also identified a small subset of patients with "missed" KD. In particular, three patients who initially had normal echocardiograms and were not treated for KD subsequently presented again and were diagnosed with $\mathrm{KD}$ with interval development of CAAs. All three patients had an illness duration of 14 days or longer, and two did not receive HDA. These cases highlight the importance of maintaining a low threshold for the diagnosis and treatment of KD based on the previously established criteria $(19,20)$, particularly in the setting of a prolonged illness course.

Our study also highlights the degree of practice variability in the management of $\mathrm{KD}$ at our center. In response to the findings presented here, we have now implemented a clinical practice guideline based on the 2017 AHA statement on KD (20).

Limitations of this study include its single-center and retrospective nature, a relatively small number of patients with CAAs, as well as loss of follow-up data. The latter is particularly true for patients with no CAAs at presentation or at 4-8 weeks, as well as for laboratory values that are occasionally not documented over time. Confounding by indication is common in retrospective studies such as this; indeed, it seems clear that the patients who received no HDA were at a later stage of disease compared with the other two groups. However, there were no clinically meaningful differences in the presenting symptoms, laboratory values, or coronary artery $Z$-scores between those patients who received 1-7 days of HDA compared with those who received $>7$ days of HDA in this study, suggesting that prolonged administration of HDA does not influence the outcomes. Of note, $34 \%$ of the patients in this study had CAAs at the time of diagnosis. This is a higher percentage than in most centers and underscores the need for a high index of suspicion for $\mathrm{KD}$ in children, especially those with incomplete $\mathrm{KD}$ who are mostly outside the expected 1-5year age range.

Overall, this study adds to a growing body of literature supporting the notion that the duration of HDA therapy does not influence meaningful long-term clinical outcomes in $\mathrm{KD}$, particularly CAA persistence. These findings highlight an opportunity to design larger, multicenter prospective trials that can provide more generalizable data regarding the interplay between HDA duration and long-term coronary artery outcomes, including the question of whether the routine use of $\mathrm{HDA}$ is necessary in the acute management of $\mathrm{KD}$.

\section{ACKNOWLEDGMENTS}

We acknowledge Michael Muradian for assisting with initial data collection.

\section{STATEMENT OF FINANCIAL SUPPORT:}

Research reported in this publication was supported by the National Center for Advancing Translational Sciences of the National Institutes of Health Award Number UL1TR000114.

\section{DISCLAIMER}

The content is solely the responsibility of the authors and does not necessarily represent the official views of the National Institutes of Health.

\section{Disclosure: The authors declare no conflict of interest.}

\section{REFERENCES}

1. Kawasaki T. Infantile acute febrile mucocutaneous lymph node syndrome with specific desquamation of the fingers and toes. Clinical observation of 50 cases. Jpn J Allerg 1967: 178-222.

2. Newburger JW, Takahashi M, Burns JC. Kawasaki disease. J Am Coll Cardiol 2016;67:1738-49.

3. Newburger JW, Takahashi M, Burns JC, et al. The treatment of Kawasaki syndrome with intravenous gamma globulin. N Engl J Med 1986;315 (6): 341-7.

4. Melish ME, Hicks RV, Reddy V. Kawasaki syndrome: an update. Hosp Pract (Hosp Ed) 1982;17:99-106.

5. Furusho K, Kamiya T, Nakano $H$, et al. High-dose intravenous gammaglobulin for Kawasaki disease. Lancet 1984;2:1055-8.

6. Newburger JW, Takahashi M, Beiser AS, et al. A single intravenous infusion of gamma globulin as compared with four infusions in the treatment of acute Kawasaki syndrome. N Engl J Med 1991;324:1633-9.

7. Dajani AS, Taubert KA, Gerber MA, et al. Diagnosis and therapy of Kawasaki disease in children. Circulation 1993;87:1776-80.

8. Asai T, Kiguciii H, Nagai Y, et al. Analysis of cardiac involvement in 29 cases with MCLS. Jpn J Pediatr 1973;26:824-36.

9. Kato H, Koike S, Yokoyama T. Kawasaki disease: effect of treatment on coronary artery involvement. Pediatrics 1979;63:175-9.

10. Yokoyama T, Kato H, Ichinose E. Aspirin treatment and platelet function in Kawasaki disease. Kurume Med J 1980;27:57-61.

11. Jacobs JC. Salicylate treatment of epidemic Kawasaki disease in New York City. Ther Drug Monit 1979;1:123-30.

12. Koren G, MacLeod SM. Difficulty in achieving therapeutic serum concentrations of salicylate in Kawasaki disease. J Pediatr 1984;105: 991-5.

13. Koren G, Rose V, Lavi S, et al. Probable efficacy of high-dose salicylates in reducingcoronary involvement in Kawasaki disease. JAMA 1985;254: 767-9.

14. Melish ME, Takahashi M, Shulman ST, et al. Comparison of low dose aspirin vs. high dose aspirin as an adjunct to intravenous gamma globulin in the treatment of Kawasaki syndrome [abstract]. Pediatr Res 1992; 31:170A.

15. Durongpisitkul K, Gururaj VJ, Park JM, et al. The prevention of coronary artery aneurysm in Kawasaki disease: a meta-analysis on the efficacy of aspirin and immunoglobulin treatment. Pediatrics 1995;96:1057-61.

16. Terai M, Shulman ST. Prevalence of coronary artery abnormalities in Kawasaki disease is highly dependent on gamma globulin dose but independent of salicylate dose. J Pediatr 1997;131:888-93.

17. Saulsbury FT. Comparison of high-dose and low-dose aspirin plus intravenous immunoglobulin in the treatment of Kawasaki syndrome. Clin Pediatr (Phila) 2002;41:597-601.

18. Shulman ST (Ed.). Management of Kawasaki syndrome: a consensus statement prepared by North American participants of the Third International Kawasaki Disease Symposium, Tokyo, Japan, December, 1988. Pediatr Infect Dis J 1989;8:663-7.

19. Newburger JW, Takahashi M, Gerber MA, et al. Council on Cardiovascular Disease in the Young; American Heart Association; 
American Academy of Pediatrics. Diagnosis, treatment, and long-term management of Kawasaki disease: a statement for health professionals from the Committee on Rheumatic Fever, Endocarditis and Kawasaki Disease, Council on Cardiovascular Disease in the Young, American Heart Association. Circulation 2004;110:2747-71.

20. McCrindle BW, Rowley AH, Newburger JW, et al. American Heart Association Rheumatic Fever, Endocarditis, and Kawasaki Disease Committee of the Council on Cardiovascular Disease in the Young; Council on Cardiovascular and Stroke Nursing; Council on Cardiovascular Surgery and Anesthesia; and Council on Epidemiology and Prevention. Diagnosis, Treatment, and Long-Term Management of Kawasaki Disease: A Scientific Statement for Health Professionals From the American Heart Association. Circulation 2017;135:e927-99.

21. Baumer JH, Love SJ, Gupta A, et al. Salicylate for the treatment of Kawasaki disease in children. Cochrane Database Syst Rev 2006;4:CD004175.

22. Colan SDNormal echocardiographic values for cardiovascular structures In: Lai WW, Cohen MS, Geva T, Mertens L eds. Echocardiography in Pediatric and Congenital Heart Disease, Appendix 1 West Sussex, UK: Wiley-Blackwell, 2009: 772-3.

23. Bland JM, Altman DG. Multiple significance tests: the Bonferroni method. BMJ 1995;310:170.

24. McCrindle BW, Li JS, Minich LLPediatric Heart Network Investigatorset al. Coronary artery involvement in children with Kawasaki disease: risk factors from analysis of serial normalized measurements. Circulation 2007;116:174-9.

25. Friedman KG, Gauvreau K, Hamaoka-Okamoto A, et al. Coronary artery aneurysms in Kawasaki disease: risk factors for progressive disease and adverse cardiac events in the US population. J Am Heart Assoc 2016;5:e003289.

26. Kuo HC, Lo MH, Hsieh KS, et al. High-dose aspirin is associated with anemia and does not confer benefit to disease outcomes in Kawasaki disease. PLoS ONE 2015;10:e0144603.

27. Dallaire F, Fortier-Morissette Z, Blais S, et al. Aspirin dose and prevention of coronary abnormalities in Kawasaki disease. Pediatrics 2017;139:e20170098.

28. Dhanrajani A, Chan M, Pau S, et al. Aspirin dose in Kawasaki diseasethe ongoing battle. Arthritis Care Res; advance online publication, 29 December 2017; https://onlinelibrary.wiley.com/doi/pdf/10.1002/acr. 23504.

29. Research Committee of the Japanese Society of Pediatric Cardiology and Cardiac Surgery Committee for Development of Guidelines for Medical Treatment of Acute Kawasaki Disease. Guidelines for medical treatment of acute Kawasaki disease: report of the Research Committee of the Japanese Society of Pediatric Cardiology and Cardiac Surgery (2012 revised version). Pediatr Int 2014;56:135-58.

30. Lee JH, Hung HY, Huang FY. Kawasaki disease with Reye syndrome: report of onecase. Zhonghua Min Guo Xiao Er Ke Yi Xue Hui Za Zhi 1992;33:67-71. 